Dieser umgelagerte allochthone Torf erreicht hier eine Mächtigkeit, die das autochthone Interglazial um ein Mehrfaches übertrifft. Ich bin mir damals über die Entstehung dieser Bildungen nicht im klaren gewesen. Es handelt sich hier ebenfalls um eine arktische Zerstörung wärme- und nachwärmezeitlicher Moore, wohl verbunden mit Palsenbildungen, wie ich sie nun im Hils nachweisen kann. Wenn diese Zerstörung, die mit der Bildung von Torfhügeln oder Palsen verbunden ist; tiefere Schichten in einem späteren Stadium ergreift, so wird im Sediment durch Auftreten von umgelagerten wärmezeitlichen und nachwärmezeitlichen Pollen, auch von Samen wie Brasenia u. a., ein zweites Wärmemaximum vorgetäuscht. P. WOLDSTEDT (1949) hat darauf schon hingewiesen. Für das Interglazial von Wallensen im Hils dürfte der Beweis für diese Erscheinung erbracht worden sein. Heute erleben wir in der Arktis nur den Abbau der in der postglazialen Wärmezeit entstandenen geringmächtigen Moore mit der Bildung von 5 und mehr Meter hohen Torfhügeln oder Palsen, wie im zentralen Lappland. Am Ende eines Interglazials muß dieses Phänomen von ganz wesentlich größerem Ausmaße gewesen sein, da hier die arktische Umlagerung mächtige Torflager erfaßte. Diese Umlagerung dürfte z. T. in Form von Mullwehen stattgefunden haben.

Literaturverzeichnis

1. IVERSEN, I.: Sekundäre Pollen als Fehlerquelle. Verh. III. Int. Quartär. Konf. Wien (1936) S. 225. 1938.

2. JESSEN, K. und MILTHERS, V.: Stratigraphical and paleontological studies of interglacial fresh water deposits in Jutland and Northwest Germany. Danm. Geol. Unders. II. 48. 1928.

3. MENZEL, H.: Beiträge zur Kenntnis der Quartärbildungen im südl. Hannover. Die Interglazialschichten von Wallensen in der Hilsmulde. - Jahrb. d. Pr. Geol. Landesanstalt. 24. 1903.

4. ORVIKU, K.: Mitteilungen über das Interglazial von Ringen-Rôngu (estnisch). Esti Loodus. Nr. 1. Dorpat-Tartu. 1939.

5. THOMSON, P. W.: Die Klima- und Waldentwicklung des von K. ORVIKU entdeckten Interglazials von Ringen beim Dorpat. - Z. deutsch. geol. Ges. 93. 1941.

6. - Beitrag zur Mikropaläontologie und Waldgeschichte des Neogens von Niedersachsen und Schleswig-Holstein. - N. Jahrb. f. Min. etc. Monatshefte. Abt. B. 1949. 1948.

7. WOLDSTEDT, P.: Über die stratigraphische Stellung einiger wichtiger Interglazialvorkommen im Randgebiet dẹr nordeuropäischen Vergletscherung. - Z . deutsch. geol. Ges. 99. 1949.

Manuskr. eingegangen Ende 1947.

\title{
Uber den heutigen Stand der Pollenuntersuchungen als Hilfsmittel der Quartärforschung
}

\author{
Von F. Firbas, Göttingen
}

Die Bedeutung der Pollenuntersuchungen für die Quartärforschung berubt im wesentlichen auf drei Umständen: Sie sind zunächst der zur Zeit wichtigste Weg für die Rekonstruktion der vergangenen Vegetation, die für den Botaniker der Gegenstand seiner Bemühungen ist, für andere Wissenschaften aber ein wichtiges Stück der Landschaft, z. B. ein Stück der Umwelt des urgeschichtlichen Menschen. Die weitgehende Abhängigkeit der Pflanzendecke vom Klima bringt es weiter mit sich, daß jede vegetationsgeschichtliche Untersuchung eine klima- 
geschichtliche Auswertung nahe legt. Und schließlich hat sich die pleistozäne und holozäne Vegetationsentwicklung als so reich gegliedert herausgestellt, daß die Zuordnung irgendwelcher Funde zu ihren einzelnen Abschnitten eine relative Altersbestimmung ermöglicht, die vor allem dort nützlich werden kann, wo andere Datierungswege versagen. Der folgende Bericht, der keinerlei Vollständigkeit erstrebt und erstreben kann und sich im wesentlichen auf die europäische Spät- und Nacheiszeit beschränken muß, ist nach diesen drei Gesichtspunkten gegliedert.

In der Rekonstruktion $d \in r$ Veget a tion stand lange Zeit im Vordergrund die spät- und postglaziale Geschichte der Wälder im extramediterranen Europa. Da es sich hierbei nur um die Erfassung weniger Gattungen mit meist sehr gut kenntlichem und sicher bestimmbarem Pollen handelt, waren in verhältnismäßig kurzer Zeit bedeutende Erfolge möglich. So stehen uns heute in Mitteleuropa Pollendiagramme aus weit über 1000 Seen und Mooren zur Verfügung, deren Ergebnisse sich statistisch verarbeiten und kartographisch (als "Pollenniederschlagskarten") darstellen lassen. Sie vermitteln ein z. T. schon sehr eingehendes Bild von der Zusammensetzung und Gliederung der Wälder während der einzelnen größeren Abschnitte der Spät- und Nacheiszeit, von denen sich etwa 10 recht gut abgrenzen lassen (1). Während der letzten Jahre sind außer in Nord- und Mitteleuropa besonders auf den britischen Inseln erfolgreiche waldgeschichtliche Arbeiten durchgeführt worden (2), und daneben erfahren wir nunmehr auch Näheres über die Waldgeschichte Frankreichs, dem dank seiner geographischen Lage für das Verständnis der nacheiszeitlichen Vegetationsentwicklung eine besondere Schlüsselstellung zukommt, das aber bisher in dieser Beziehung noch sehr wenig erschlossen war. Die wichtigste Frage, die man hier stellen möchte - nämlich nach dem Verlauf und der Zusammensetzung der glazialen Waldgrenze — läßt sich allerdings vorläufig immer noch nicht beantworten (3). Úber die in Rußland erzielten neueren Fortschritte ist Verf. leider noch kaum unterrichtet.

Wir stehen aber heute in einer Periode eines systematischen Ausbaus der Pollenuntersuchungen, der weit über ihre Anwendung in der jüngeren Waldgeschichte hinausgeht und die Erfassung des Gesamtpollengehalts der Ablagerungen zum Ziel hat. Dieser Ausbau vollzieht sich auf drei Wegen:

Erstens in der zunehmenden Anwendung von Pollenuntersuchungen im Vorpleistozän, besonders im Tertiär. Darüber ist hier nicht zu berichten (4).

Zweitens in der zunehmenden Anwendung der Pollenanalyse in anderen Florengebieten. Neben einer großen Zahl von Untersuchungen der nacheiszeitlichen Vegetationsentwicklung in den Vereinigten Staaten und in Kanada verfügen wir z. B. über solche aus Feuerland und Neuseeland und neuerdings vor allem über umfassend begründete Pollenuntersuchungen an den Gebirgsmooren der Regenwaldstufe der Hawaiischen Inseln. Doch kann auch darauf hier nicht näher eingegangen werden (5).

Drittens handelt es sich im glazialen und postglazialen Mitteleuropa um den Ausbau der Bestimmung der sogenannten Nichtbaumpollen, worunter man alle Pollenkörner außer den bekannten Gehölzpollen versteht. Die Nichtbaumpollen haben zunächst Beachtung gefunden, weil sich aus ihrem Mengenverhältnis zu den Baumpollen Schlüsse auf den Grad der Waldbedeckung einer Landschaft ziehen lassen. Auf diese Weise wurde es möglich, in den Pollendiagrammen den Zeitpunkt der ersten Wiederbewaldung zu erfassen und waldlose Glazial- und Spätglazialfloren aufzufinden, die sich bisher dem Nachweis entzogen hatten, z. B. auch solche in den heute wärmsten Landstrichen des innerböhmischen Elb- 
tals. Auf Grund dieser Untersuchungen wissen wir heute mit Sicherheit, daß Mitteleuropa zwischen den Alpen und dem nordischen Inlandeis während der letzten Eiszeit so gut wie vollständig waldlos gewesen sein muß (6).

Aber schon 1931 versuchten OVERBECK und SCHMITZ durch eine qualitative Analyse der Nichtbaumpollen auch die Geschichte anderer Pflanzengesellschaften aufzudecken, nämlich der nordwestdeutschen Zwergstrauchheiden und der die Küste begleitenden Halophytengesellschaften. Einige Jahre später gelang der pollenanalytische Nachweis des Getreidebaus, und durch diesen und die Bestimmung des Pollens einiger den Menschen begleitender Unkräuter, vor allem der Wegerich-(Plantago-)Arten, spiegelt sich heute auch die Geschichte der menschlichen Siedlungen, so weit sie mit Ackerbau verbunden waren, in den Pollendiagrammen wider (7). Das hat in Landschaften, die erst in bekannter, historischer Zeit besiedelt worden sind, Bedeutung für die Datierung der Diagramme. Es gestattet weiter, die Veränderungen der Vegetation unter dem Einfluß der Besiedlung zu verfolgen und ist schließlich auch verwendbar für die Klärung der älteren Siedlungsgeschichte selbst. So ließ sich erstmals im Untereichsfeld, später im Federseegebiet eine mehrfache Diskontinuität der mit Ackerbau verbundenen Besiedlung nachweisen (8).

Heute wendet sich nun das Interesse bei der Untersuchung der Nichtbaumpollen vor allem der näheren Bestimmung der Glazialvegetation zu, besonders dem Anteil, den "Tundra" und "Steppe“ an ihr nahmen. Seit A. NEHRING's berühmten Untersuchungen (1875 ff.) kennt man allgemein die Steppenkomponente der glazialen Fauna, schon vorher war aus den zerstückelten Verbreitungsgebieten von Steppenpflanzen auf eine frühere Steppenzeit geschlossen worden. Das Vorkommen von Steppenpflanzenkolonien in den Zentralalpentälern lehrte, daß wenigstens ein Abschnitt dieser Steppenzeit postglazial (i. w. S.) sein muß. Aber in merkwürdigem Gegensatz zur Fauna blieb der paläontologische Nachweis glazialer Steppenpflanzen auffällig bescheiden, wenn auch Funde wie jene von Crambe tataria bei Przemysl und neuerdings von Alyssum saxatile (Arduini) bei Tarnow, also in galizischen Glazialfloren, von besonderem Interesse sind (9). Nun haben aber IVERSEN und WELTEN fast gleichzeitig zeigẹn können, daß Helianthemum cf. alpestre, eine Leitart trockener subalpiner Matten, an der waldlosen Vegetation des älteren Spätglazials einen besonders hohen Anteil hatte, und ein noch viel besserer Zeiger für eine Trockenheit fordernde und in diesem Fall wohl steppenähnliche Vegetation ist das reichliche Vorkommen des lange Zeit mit Salix verwechselten Artemisia-Pollens, der z. B. am Bodensee bis $19^{0} \%$ des Gesamtpollens ausmachen kann (10). Allerdings kommt Artemisia campestris ssp. borealis auch in der arktischen Tundra vor. Aber nach den bisherigen Analysen, die aus dem Verbreitungsgebiet $\operatorname{der} A$. borealis stammen, ist die Häufigkeit des Artemisia-Pollens dort verschwindend gering (unter 1\%) und mit der glazialen, bzw. spätglazialen in Mitteleuropa nicht zu vergleichen (11). So dürfen wir erwarten, daß sich unser Bild von der eiszeitlichen Pflanzendecke im Laufe der nächsten Jahre wesentlich bereichern wird.

Der dem Gebiet Fernstehende mag sich freilich wundern, daß der pollenanalytische Nachweis vegetationsgeschichtlich so wichtiger Pflanzen erst so spät und nur schrittweise gelingt. Die Ursache hierfür liegt darin, daß die Morphologie und Systematik der Pollen- und Sporenformen ein sehr schwieriges Gebiet ist, das lange Zeit nicht die genügende Beachtung fand. Immer noch fehlt uns eine vollständige und kritische europäische Pollenflora. Sie kann nur auf Grund sorgfältiger Monographien im Laufe der Zeit ausgebaut werden; übereilte „Bestimmungsbücher" sind wertlos. Doch belebt sich das. Interesse an der Pollen- 
systematik zusehends, nicht zuletzt dank der unermüdlichen Tätigkeit G. E. ERDMAN's (12). Schweden hat vor kurzem in Bromma (Stockholm) auch ein eigenes Institut für Pollenforschung (Palynologie) gegründet. Außerdem wird von IVERSEN, TROELS-SMITH, FAEGRI u. a. in Dänemark und Norwegen auf diesem Gebiet intensiv gearbeitet. Von den Fortschritten der qualitativen Pollenanalyse darf man wohl auch weitere Aufschlüsse über den Ausklang der pliozänen Flora in den Interglazialen und damit neue Anhaltspunkte für deren Altersstellung erwarten.

Wenden wir uns nun kurz dem Nachweis von Klimä nde rungen zu, so haben sich innerhalb des Zeitraums vom letzten Eishochstand der Würmeiszeit (Pommersches Stadium) bis zur Gegenwart zwei große Wärmeschwankungen herausgestellt: das spätglaziale Alleröd-Interstadial und die postglaziale Wärmezeit.

$\mathrm{Zu}$ einer Übersicht über die spätglazialen Verschiebungen der Waldgrenze und die ihnen zugrunde liegenden wahrscheinlichen Temperaturveränderungen, die an anderer Stelle gegeben wurde (13), sei hier nur ergänzend hinzugefügt, daß G. DUBOIS auch über einen wahrscheinlichen Nachweis der Allerödschwankung im französischen Zentralplateau, an zwei Stellen in 950 und $1100 \mathrm{~m}$ Höhe im Massiv von Cantal, berichten konnte. Es ist bezeichnend, daß die hier dem Alleröd zugeordneten Schichten schon erhebliche Mengen von Eichenpollen enthalten, der später wieder verschwindet. Weiter konnte W. PENNINGTON in Mittelengland in dem See von Windermere, der am Fuß der bis $978 \mathrm{~m}$ ansteigenden Cambrian Mountains liegt, ein Spätglazialprofil aufdecken, in dem die Allerödschwankung durch Grobdetritusgyttjen, die jüngere Dryaszeit aber nochmals durch Bändertone, Zeugen einer neuerlichen Vergletscherung des Gebirges, vertreten ist. Sie umfassen nach vorläufiger Zählung etwa 400-500 Jahresschichten. Das stützt die übliche Zuordnung zu dem Eisstand an den Salpausselkä (14). Hatten sich subarktische Birken- und Kiefernwälder während der Allerödzeit bis ins Ostseegebiet ausgebreitet, so muß der nachfolgende Klimarückschlag der jüngeren Tundrenzeit den geschlossenen Wald wieder weit nach Süden zurückgedrängt haben. Er scheint sich als solcher nur in den wärmsten Tieflagen der Mittelgebirgslandschaften erhalten zu haben wie im Oberrheinischen Tiefland, in Innerböhmen u. a. Weite Landschaften Mitteleuropas wurden waldlos oder zu einer Art „Waldtundra“. Die alpine Waldgrenze sank wieder um einige $100 \mathrm{~m}$ auf Höhenlagen von $800-1100 \mathrm{~m}$ unter ihrem heutigen Stand herab.

Festigt sich unser Wissen von den spätglazialen Klimaschwankungen erst jetzt, so ist die postglaziale Wärmezeit seit langem erwiesen und anerkannt. Trotzdem knüpfen sich auch an sie noch mancherlei Fragen. Sie betreffen ihre nähere klimatische Charakteristik, die genauere Bestimmung ihres Anfangs, ihres Verlaufs und ihres Endes. Hier kann darauf nicht näher eingegangen werden. An anderer Stelle wurde z. B. gezeigt, daß der seit langem erschlossene höhere Stand der Waldgrenze im Riesengebirge, mit dem eine Bedeckung der Kammregion bis oberhalb $1500 \mathrm{~m}$, sehr wahrscheinlich mit subalpinem Buchengehölz, verbunden war, nicht - wie bisher angenommen - nur bis in den Beginn des Subatlantikums, sondern bis in die erste Zeit der deutschen Kolonisation angedauert hat, nämlich mindestens bis ins 14. Jahrhundert. Erst im Laufe des 15. und 16. Jahrhunderts kann der Übergang zu der heutigen (nach Abwägung wirtschaftlicher Einflüsse als natürlich anzusehenden) Lage erfolgt sein. Das steht in guter Übereinstimmung, z. B. zu dem, was wir über die historischen Gletscherstände in den Alpen wissen (15). Besteht also zwischen dem bekannten Temperaturverlauf des Spätglazials und der Strahlungskurve ein 
auffälliger Gegensatz (13), so paßt der Nachweis eines allmählichen Ausklangs der postglazialen Wärmezeit auch noch in der sogenannten "Nachwärmezeit" recht gut zu der während der letzten Jahrtausende weiter fortschreitenden Strahlungsabnahme.

Einen groß angelegten und umfassend begründeten Überblick über die Bedeutung der Pollenuntersuchungen für die Klimageschichte der Erde hat 1944 L. v. POST gegeben. Sein Grundgedanke ist der Nachweis der „Revertenz", wie sie sich in allen bisher untersuchten Vegetations- und Klimagebieten der Erde aus den auffälligen regionalen Parallelen im Kurvenverlauf der Pollendiagramme ergibt: Im letzten, etwa 2-3 Jahrtausende umfassenden Abschnitt der Klimageschichte der Erde (III) läßt sich in der Vegetationsentwicklung und damit offenbar auch im Klima eine neuerliche Annäherung an die Zustände erkennen, die im frühen Postglazial (I) bestanden haben. Dazwischen liegt ein mehrere Jahrtausende umfassender mittlerer Abschnitt (II), der uns als „postglaziale Wärmezeit" mit einer Polwärtsverschiebung der gemäßigten Waldgürtel um etwa 5 Breitengrade bekannt ist, in anderen Klimagebieten aber auch durch Verschiebungen der Niederschlagsverhältnisse zum Ausdruck kommen kann. So herrschte in den untersuchten höheren Lagen der Hawaiischen Inseln während I eine trockene subalpine Vegetation, während II montaner Regenwald. Während III macht sich neuerlich die Ausbreitung einer zwar etwas anders gearteten, aber deutlich trockeneren subalpinen Vegetation geltend. Ähnliches gilt für Neuseeland. Es muß betont werden, daß die Vegetations- und wohl auch die Klimaverhältnisse in den meisten Gebieten während III andere waren als während I, aber eine gewisse Annäherung, eine „Revertenz" ist trotzdem, vor allem im Vergleich zu II, unverkennbar. Diese Gedanken v. POST's werden die Forschung in den nächsten Jahren sicher noch mehr bestimmen, als sie das schon bisher taten. Es eröffnet sich jetzt durch die Untersuchungen in verschiedenen Klimagebieten der Erde die Möglichkeit, Einblick in jene Änderungen im Zirkulationssystem der Atmosphäre zu gewinnen, die den postglazialen Klimaverlauf in den verschiedenen Gebieten zwar jeweils anders, aber doch entsprechend und in enger ursächlicher Verknüpfung bestimmt haben.

Zur Frage der Altersbestimmung mit Hilfe von Pollenuntersuchungen sei nochmals wiederholt, daß die Pollendiagramme der meisten mitteleuropäischen Landschaften im Laufe des Spät- und Postglazials die Unterscheidung von 8-10 Abschnitten möglich machen, die durch ihre Pollenführung so gut gekennzeichnet sind, daß eine sichere Zuweisung von Funden unbekannten Alters zu ihnen, wenn nicht schon auf Grund einzelner Pollenspektren, so doch auf Grund längerer Diagrammausschnitte möglich wird. Darüber hinaus zu gehen, ist hingegen schwierig. Es sei an einer einfachen Überlegung gezeigt. Die Zeit seit dem frühpostglazialen Haselmaximum bis zur Gegenwart beträgt etwa 8000 Jahre, d. h. 80 Jahrhunderte. Um durchgehend mit einer Genauigkeit von einem Jahrhundert datieren zu können, müßten wir also in den Pollendiagrammen diesen Zeitraum in 80 Abschnitte teilen und jeden einzelnen dieser $\mathrm{Ab}$ schnitte von den Nachbarabschnitten unterscheiden können. Jeder Blick auf ein Pollendiagramm mit seinen methodisch bedingten Zufallsschwankungen lehrt, daß dies auch für längere Zeitabschnitte, z. B. von 200-300 Jahren, in der Reged nicht möglich sein wird. Nur zu gewissen Zeiten, etwa während der Erstausbreitung der Buche oder dort, wo in jungbesiedelten Gebieten die Getreidekurve mit der bekannten historischen Siedlungsgeschichte verknüpft werden kann, liegen die Dinge etwas günstiger. Aber Altersunterschiede von nur einem oder einigen wenigen Jahrhunderten können darüber entscheiden, ob ein Fund z. B. 
noch ins Neolithikum oder schon in die Bronzezeit zu stellen ist. Die Vorsicht und Kritik, die hier unbedingt notwendig ist, ist nicht immer geübt worden und wird es auch heute noch nicht in allen Fällen. So erfreulich es daher für den Vegetationsgeschichtler ist, wenn er anderen Wissenschaften, besonders der Urgeschichte, nützlich sein kann, so besteht heute zweifellos die Gefahr, daß die Mittel, die ihm zur Verfügung stehen und die bei kritischer Verwendung sicherlich wichtige Datierungen möglich machen, überschätzt werden.

Um so größer ist freilich auch der Anreiz, eine Verfeinerung der Altersbestimmungen $\mathrm{zu}$ erstreben. Sie wird heute auf vierfache Weise verfolgt:

Erstens durch den Nachweis kleinerer Klimaschwankungen und ihre sichere Verknüpfung von Landschaft zu Landschaft. Hier stehen neben den Vorgängen, die in untergeordneten, aber gesetzmäßigen und von den methodischen Schwankungen abtrennbaren Verschiebungen des Pollengehalts zum Ausdruck kommen, jene Änderungen der Feuchtigkeitsverhältnisse zur Verfügung, die das Wachstum der Moore beeinflußt haben. In Südschweden hat die wichtigsten GRANLUND in seinem System der Rekurrenzflächen (Zersetzungskontakte des Hochmoortorfs) erfaßt, von denen die dritte dem klassischen Grenzhorizont C. A. WEBER's entspricht (16). Es ist eine wichtige, aber nur durch sehr eingehende monographische Untersuchungen lösbare Aufgabe, den Geltungsbereich der durch die Rekurrenzflächen angezeigten Klimaveränderungen in Europa zu bestimmen. Aus Dänemark und England liegen z. B. neue positive Beiträge hierzu vor (17). Die von OVERBECK und SCHNEIDER eingeführte regelmäßige Anwendung kolorimetrischer Untersuchungen zur Bestimmung der Humifizierung des Torfes dürfte auf dem Wege zu einer größeren Exaktheit auf diesem Gebiete noch von besonderem Nutzen werden (18).

Ein weiterer Weg zum Ausbau pollenanalytischer Altersbestimmungen ist die Auswertung der hie und da vorhandenen Jahresschichtung pollenführender Ablagerungen. Er ist schon 1925 von GIESENHAGEN an dem interglazialen Kieselgurlager von Neu-Ohe begangen worden. SANDEGREN und später FROMM haben die für die Chronologie des Postglazials entscheidenden gebänderten Sedimente des Ragundasees und Ångermanelfs pollenanalytisch untersucht, und neben Funden in der Kieler Föhrde (TAPFER) liegt ein eingehender Versuch aus dem Faulenseemoos im Gebiet des Thuner Sees durch WELTEN vor (19). Von den sicher datierten Diagrammen FROMM's abgesehen, steckt dieses Verfahren aber noch in den Anfängen. Dies gilt noch mehr von einem dritten, nämlich einer Verknüpfung der Pollendiagramme mit der Jahrringchronologie, die seit einer Reihe von Jahren für Deutschland durch Br. HUBER ausgebaut wird (20). Allen diesen Möglichkeiten ist eines gemeinsam: daß nur ein sehr großer Aufwand an Zeit und Arbeitskraft und nur peinlichste, unvoreingenommene Genauigkeit und Geduld zum Ziele führen können. Von der Datierung der Interglaziale auf Grund ihrer Pollendiagramme soll hier nicht die Rede sein. P. WOLDSTEDT hat mit seinen Mitarbeitern gerade bei der Hannoverschen Tagung hiefür verschiedene neue überzeugende Beispiele beigebracht. Schließlich ist vor kurzem auch noch die Aussicht hinzugekommen, Torfe und sonstige organische Reste mit Hilfe ihres Gehalts an radioaktivem Kohlenstoff (C14) datieren zu können (21).

Die Pollenforschung mochte in den Jahren, in denen sie sich fast ganz mit der postglazialen Waldgeschichte beschäftigt hat, dem Fernstehenden fertig und einförmig erscheinen. Tatsächlich ist ein ständiger methodischer Ausbau vor sich gegangen, der ihr Anwendungsgebiet wesentlich erweitert hat, noch lange nicht abgeschlossen ist und daher weitere Erfolge verspricht. 
Schriftennach weis

(1) FIRBAS, F., Spät- und nacheiszeitliche Waldgeschichte Mitteleuropas nördlich der Alpen. Jena 1949.

(2) Besonders von H. GODWIN und seinen Schülern sowie in Irland von Kn. JESSEN und G. F. MITCHELL, vgl. die Sammelreferate von F. FIRBAS in Naturwissenschaften, 31, 1943, 69-72 (für Nordeuropa) und a. gl. O. 34, 1947, 252-256 (für die Britischen Inseln), weiter Proc. Roy. Irish Acad. 52/B, Nr. 6, 1949.

(3)

DUBOIS, G., Vegetatio 1, 1948, 43-50; LEMEE, G., Ann. Biol., 24, 1948, 49-75, und FIRBAS in Fortschritte der Botanik, 12, 1949.

(4) Vgl. z. B. THIERGART, F., Die Mikropaläontologie als Pollenanalyse im Dienst der Braunkohlenforschung, Stuttgart 1940, und die dort besprochenen Arbeiten. Seit mehreren Jahren hat P. W. THOMSON auf diesem Gebiet erfolgreich gearbeitet, vgl. verschiedene Arbeiten in Geol. Jahrb. (Hannover), 65, 1950 und in Paläontographica, 90/B, 1950; außerdem KIRCHHEIMER, POTONIE, RUDOLPH u. a.

(5) POST L. v., The prospect for pollen analysis in the study of the earth's climatic history. New Phytologist, 45, 1946, 193-217 (Ubersetzung aus Ymer 1944); SELLING, O. H., B. P. Bishop Museum Spec. Publ. 39, Honolulu (Hawaii), $1948,1-154$.

(6) FIRBAS, F., Naturwissenschaften, 27, 1939, 81-89, 104-108.

(7) OVERBECK, F. und SCHMITZ, H., Mitt. Provinzialst. f. Naturdenkmalpfl. Hannover, 3, 1931, 1-179; FIRBAS, F., Zeitschr. f. Botanik, 31, 1937, 447-478; IVERSEN, J., Danm. Geol. Unders. Kobenhavn, II/66, 1941, 7-68.

(8) STEINBERG, K., Hercynia (Halle), 3, 1944, 529-587; MÚLLER, I., Planta 35, 1947, 70-87.

(9) KULCZYNSKI, St., Acta Soc. Bot. Polon., 9, 1932; KLIMASZEWSKI, M. und SZAFER, Wl., Starunia (Krakow), 19, 1945, 1-34.

(10) IVERSEN, J., Geol. Fören. Förh. Stockholm, 66, 1944, 774-776; WELTEN, M., Veröff. d. Geobot. Inst. Rübel, Zürich, 21, 1944, 1-201; FIRBAS, F., Biol. Zbl., 67, 1948, 17-22; ERDTMAN, G. E., Svensk. Bot. Tidskr., 40, 1946, 293-304.

DEGERBOL, L. und IVERSEN, J., Danmarks Geol. Unders. Kobenhavn, II/73, 1945, 1-62; WENNER, C. G., Geografiska Annaler, 1947, 1-241.

(12) ERDTMAN, G. E., An Introduction to Pollen Analysis, Waltham, Mass. 1943, 1-239, und verschiedene Beiträge in Botan. Notiser (Lund) 1944-1946 FAEGRI, Kn., a. J. IVERSEN, Text-book of modern pollenanalysis. Copenhagen 1950.

(13) FIRBAS, F., Naturwissenschaften, 34, 1947, 114-118.

(14) DUBOIS, G., \& C., C.R.S. de la Soc. Géol. de France, 1944, 46-48 und 61-68; PENNINGTON, W., Phil. Transact. Royal Soc. London, B/596, 233, 1947, $137-175$.

(15) FIRBAS, F., und LOSERT, H., Planta 36, 1949, 478-506.

(16) GRANLUND, E., Sverig. Geol. Undersökn. Arsbok, 26, 1932, 1-193.

(17) JESSEN, K., Acta Archaeol. Kobenhavn, 16, 1-3, 1945, 67-91; HARDY, E. M., New Phytologist, 38, 1939, 364-396.

(18) OVERBECK, Fr., Planta, 35, 1947, 1-56.

(19) FROMM, E., Geol. Fören. Förhandl. Stockholm, 60, 1938, 365-381; TAPFER, E., Geol. d. Meere und Binnengewässer, 4, 1940, 113-244; WELTEN, M., s. u. (10).

(20) HUBER, Br., Mitt. d. Akad. d. Dtsch. Forstwissensch., 1, 1941, 110-125.

(21) LIBBY u. a. in Science, 109, 1949. 